09,12

\title{
Исследование электронной структуры и спектров оптического поглощения икосаэдрического золотого фуллерена $\mathrm{Au}_{42}$
}

\section{(C) Г.И. Миронов}

Марийский государственный университет, Йошкар-Ола, Россия

E-mail: mirgi@marsu.ru

Поступила в Редакцию 10 октября 2020 г.

В окончательной редакции 10 октября 2020 г.

Принята к публикации 16 октября 2020 г.

\begin{abstract}
Электронная структура молекулы золотого фуллерена $\mathrm{Au}_{42}$ изучается в рамках гамильтониана Хаббарда. Получены выражения для Фурье-образов функций Грина, полюса которых определяют энергетический спектр рассматриваемого нанокластера. Энергетический спектр $\mathrm{Au}_{42}$ исследуется в сравнении со спектром икосаэдрического золотого фуллерена $\mathrm{Au}_{32}$. Энергетический спектр свидетельствует о полупроводниковом состоянии золотого фуллерена $\mathrm{Au}_{42}$. Приведена плотность электронных состояний, пики которой соответствуют особенностям Ван Хова. Приведены спектры оптического поглощения нейтрального и отрицательно заряженного фуллерена $\mathrm{Au}_{42}$, энергия первого прямого оптического перехода отрицательно заряженного иона золотого фуллерена $\mathrm{Au}_{42}^{-}$равна $0.985 \mathrm{eV}$, находится в ближней инфракрасной области. Показана возможность применения исследуемого нанокластера из атомов золота для диагностики и лечения раковых заболеваний.
\end{abstract}

Ключевые слова: золотой фуллерен, спектр оптического поглощения, плотность электронных состояний, антикоммутаторная функция Грина, энергетический спектр.

DOI: 10.21883/FTT.2021.02.50480.216

\section{1. Введение}

Золотой фуллерен $\mathrm{Au}_{42}$ имеет идеальную икосаэдрическую структуру, он будто построен из икосаэдрического углеродного фуллерена $\mathrm{C}_{80}$ в качестве шаблона. Фуллерен $\mathrm{Au}_{42}$ состоит из 12 вершин, 30 ребер и 20 треугольных граней (карат). Каждое ребро имеет три атома золота, а каждая треугольная грань - шесть атомов $\mathrm{Au}$ (см. рис. 1).

Интерес к золотым фуллеренам и к золотому фуллерену $\mathrm{Au}_{42}$ в частности обусловлен особенными физическими и химическими свойствами золотых фуллеренов, как бы промежуточных между свойствами отдельного атома $\mathrm{Au}$ и объемного твердотельного материала из атомов золота, которые представляют большой интерес для практического применения в области катализа [1,2], материаловедения [3], медицине [4-6].

Согласно [7-10] фуллерен $\mathrm{Au}_{42}$ имеет небольшую энергетическую щель между самой высокой занятой молекулярной орбиталью (НОМО) и самой низкой незанятой молекулярной орбиталью (LUMO), равную $0.4 \mathrm{eV}$ при использовании популярного функционала ВР86 [11] и $0.9 \mathrm{eV}$ при B3LYP [12] вычислениях. Орбиталь НОМО является пятикратно вырожденной, а LUMO - четырехкратно вырожденной. Если взять икосаэдрический фуллерен $\mathrm{Au}_{32}$ [13], энергетическая щель между LUMO и НОМО равна 1.7 и $2.5 \mathrm{eV}$ при вычислениях с функ- ционалами ВР86 и РВЕ0 [14] соответственно. Кратность вырождения и HOMO и LUMO оказалась равной четырем [13]. Поэтому делается вывод, что фуллерен

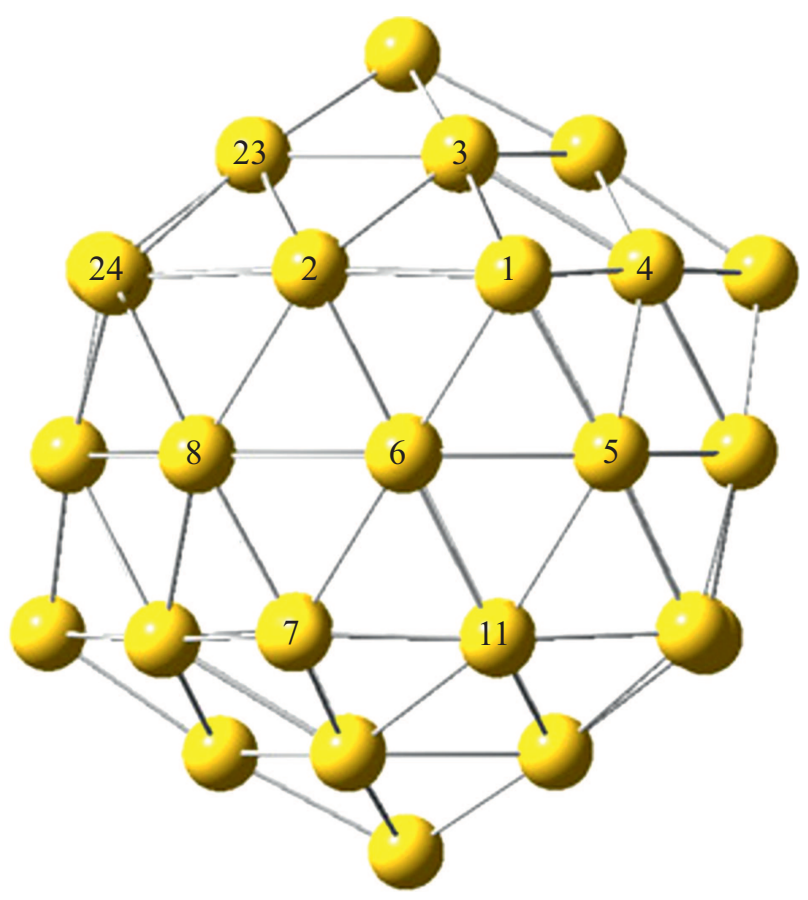

Рис. 1. Модель молекулы фуллерена $\mathrm{Au}_{42}$. 
$\mathrm{Au}_{42}$ не так химически стабилен, как икосаэдрический фуллерен $\mathrm{Au}_{32}$. В [1] подчеркивается, что фуллерен $\mathrm{Au}_{42}$ является стабильным, обсуждаются механизмы участия фуллерена $\mathrm{Au}_{42}$ в химических реакциях как катализатора.

\section{2. Теоретическая модель}

Целью настоящей работы является теоретическое изучение электронной структуры фуллерена из атомов золота $\mathrm{Au}_{42}$ с учетом того, что $\mathrm{Au}_{42}$ является системой с сильными корреляциями, при этом определяющую роль играют $d$-электроны, так как уровни энергии $d$-электронов оказываются выше спектра $s$-электронов $[15,16]$, в электронном транспорте участвуют только $d$-электроны.

Для изучения квантовых систем, в которых определяющую роль играют $d$-электроны, более полувека назад была предложена модель Хаббарда [17]. Следует подчеркнуть, что за 30 лет до работы Хаббарда предлагалась более общая модель [18] Шубина-Вонсовского, модель Хаббарда является частным случаем модели, предложенной в [18]. В рамках гамильтониана Хаббарда атом переходного металла заменяется моделью, в которой вокруг положительно заряженного иона движется электрон. В зависимости от того, насколько этот электрон локализован или делокализован, получается фазовый переход металл-диэлектрик, удается объяснить наиболее важные свойства переходных металлов, а также понять причины особенностей свойств наноструктур $[19,20]$.

Гамильтониан модели Хаббарда рассматриваемой молекулы фуллерена из 42 атомов золота имеет вид

$$
\begin{gathered}
\hat{H}=\hat{H}_{0}+\hat{V}, \\
\hat{H}_{0}=\sum_{\alpha, f=1}^{42} \varepsilon n_{f \sigma}+\sum_{\sigma, f \neq l} B_{f l}\left(a_{f \sigma}^{+} a_{l \sigma}+a_{l \sigma}^{+} a_{f \sigma}\right), \\
\hat{V}=U \sum_{f=1}^{42} \hat{n}_{f \uparrow} \hat{n}_{f \downarrow},
\end{gathered}
$$

где $a_{j \sigma}^{+}, a_{j \sigma}-$ Ферми-операторы рождения и уничтожения электронов на узле $j(j=f, l)$ золотого фуллерена с проекцией спина $\sigma(\sigma=\uparrow, \downarrow) . \hat{n}_{j \sigma}=a_{j \sigma}^{+} a_{j \sigma}-$ оператор числа частиц, $\varepsilon-$ собственная энергия электрона, $U-$ энергия кулоновского взаимодействия электронов с противоположно ориентированными проекциями спинов на одной орбитали, $B_{f l}=B(f-l)-$ интеграл переноса электрона с узла на соседний узел молекулы фуллерена.

Уравнения движения для операторов рождения электронов в гейзенберговском представлении имеют вид

$$
\begin{aligned}
& \text { ( } \tau=i t-\text { мнимое время): } \\
& \frac{d}{d \tau} a_{1 \uparrow}^{+}=\varepsilon a_{1 \uparrow}^{+}+B\left(a_{2 \uparrow}^{+}+a_{3 \uparrow}^{+}+a_{4 \uparrow}^{+}+a_{5 \uparrow}^{+}+a_{6 \uparrow}^{+}\right) \\
& +U \hat{n}_{1 \downarrow} a_{1 \uparrow}^{+}, \\
& \frac{d}{d \tau} a_{2 \uparrow}^{+}=\varepsilon a_{2 \uparrow}^{+}+B\left(a_{1 \uparrow}^{+}+a_{3 \uparrow}^{+}+a_{6 \uparrow}^{+}+a_{8 \uparrow}^{+}+a_{23 \uparrow}^{+}\right. \\
& \left.+a_{24 \uparrow}^{+}\right)+U \hat{n}_{2 \downarrow} a_{2 \uparrow}^{+}, \\
& \frac{d}{d \tau} a_{42 \uparrow}^{+}=\varepsilon a_{42 \uparrow}^{+}+B\left(a_{29 \uparrow}^{+}+a_{32 \uparrow}^{+}+a_{35 \uparrow}^{+}+a_{38 \uparrow}^{+}\right. \\
& \left.+a_{41 \uparrow}^{+}\right)+U \hat{n}_{42 \downarrow} a_{42 \uparrow}^{+} \cdot
\end{aligned}
$$

Система сорока двух операторных уравнений (4) решается в рамках приближения статических флуктуаций [16].

\section{3. Результаты и их обсуждение}

Методика решения систем уравнений движения типа (4), вычисления Фурье-образа антикоммутаторной функции Грина применительно к наносистемам подробно приведена в работах $[16,19,20]$, поэтому сразу приведем формулу для Фурье-образа антикоммутаторной функции Грина фуллерена $\mathrm{Au}_{42}$ для атома в центре правильного пятиугольника, составленного из треугольников (см. рис. 1):

$$
\begin{aligned}
& \left\langle\left\langle a_{1 \uparrow}^{+} \mid a_{1 \uparrow}\right\rangle\right\rangle_{E}=\frac{i}{2 \pi} \sum_{n=1}^{2}\left\{\frac{0.0448}{E-\varepsilon_{n}+2.534 B}\right. \\
& +\frac{0.1080}{E-\varepsilon_{n}+0.517 B}+\frac{0.0555}{E-\varepsilon_{n}-3.051 B} \\
& +\frac{0.0407}{E-\varepsilon_{n}+2.3917 B}+\frac{0.0947}{E-\varepsilon_{n}+1.5211 B} \\
& +\frac{0.0843}{E-\varepsilon_{n}-1.1556 B}+\frac{0.0303}{E-\varepsilon_{n}-4.7572 B} \\
& \left.+\frac{0.0097}{E-\varepsilon_{n}-5.7417 B}+\frac{0.0320}{E-\varepsilon_{n}+1.7417 B}\right\} .
\end{aligned}
$$

Приведем Фурье-образ функции Грина для атома, находящегося в центре правильного шестиугольника, 
составленного из правильных треугольников:

$$
\begin{aligned}
& \left\langle\left\langle a_{2 \uparrow}^{+} \mid a_{2 \uparrow}\right\rangle\right\rangle_{E}=\frac{i}{2 \pi} \sum_{n=1}^{2}\left\{\frac{0.0654}{E-\varepsilon_{n}+2.534 B}\right. \\
& +\frac{0.0401}{E-\varepsilon_{n}+0.517 B}+\frac{0.0611}{E-\varepsilon_{n}-3.051 B} \\
& +\frac{0.0337}{E-\varepsilon_{n}+2.3917 B}+\frac{0.0121}{E-\varepsilon_{n}+1.5211 B} \\
& +\frac{0.0163}{E-\varepsilon_{n}-1.1556 B}+\frac{0.0379}{E-\varepsilon_{n}-4.7572 B} \\
& +\frac{0.0128}{E-\varepsilon_{n}-5.7417 B}+\frac{0.0039}{E-\varepsilon_{n}+1.7417 B} \\
& \left.+\frac{0.0667}{E-\varepsilon_{n}-B}+\frac{0.0667}{E-\varepsilon_{n}+B}+\frac{0.0379}{E-\varepsilon_{n}+2 B}\right\} \text {. }
\end{aligned}
$$

В формулах (5) и (6)

$$
\varepsilon_{n}=\left\{\begin{array}{ll}
\varepsilon, & n=1 \\
\varepsilon+U, & n=2
\end{array} .\right.
$$

Фурье-образы антикоммутаторных функций Грина для других атомов будут совпадать либо с выражением (5), либо с формулой (6). Полюса функций Грина определяют энергетический спектр, эти спектры приведены на рис. 2, $a$ и $b$. На рис. 2, $a$ приведены уровни энергии для атома, расположенного в центре правильного пятиугольника, составленного из правильных треугольников, при значениях параметров исследуемой системы $\varepsilon=-2.85 \mathrm{eV}, B=-1 \mathrm{eV}, U=8.85 \mathrm{eV}$, на рис. $2, b-$ для атома, находящегося в центре правильного шестиугольника при тех же значениях параметров. Если в случае первого атома электроны могут располагаться только на 18 уровнях энергии (9 верхних уровней энергии образуют верхнюю хаббардовскую подзону, которую мы можем назвать и зоной проводимости, 9 нижних уровней энергии образуют нижнюю хаббардовскую подзону (валентную зону). Числа, приведенные на левой стороне от уровней энергии, говорят о следующем: например, в случае первого атома электрон на самом верхнем уровне энергии $8.534 \mathrm{eV}$ может находиться с вероятностью 0.045, тогда как в случае атома № 2 на этом же уровне энергии $8.534 \mathrm{eV}$ электрон может находиться с чуть большей вероятностью, равной 0.065. На следующем уровне энергии $8.392 \mathrm{eV}$ электрон в центре пентагона может находиться с вероятностью 0.041 , а в атоме, расположенной в центре гексагона, электрон может находиться с меньшей вероятностью, равной 0.034 . На рис. 2, $b$ справа есть уровень (третий сверху) энергии $8 \mathrm{eV}$, на ней электрон может находиться с вероятностью 0.083 , тогда как в случае атома, находящегося в центре пентагона, такой уровень энергии отсутствует это означает, что в случае атома № 1 электрон на уровне энергии $8 \mathrm{eV}$ может находиться лишь с вероятностью, равной нулю.
На рис. 3 приведен вид энергетического спектра молекулы фуллерена $\mathrm{Au}_{42}$. На рисунке над или под линиями, в четырех случаях прямо на линиях приведены значения энергии в электронвольтах, значения орбитального квантового числа $l$, кратности вырождения уровней энергии, с правой стороны приведены обозначения состояний, соответствующих уровням энергии. Символы $a, t_{1}, t_{2}, g, h$ характеризуют соответственно степени вырождения $1,3,3,4,5$, правый нижний индекс $g$ свидетельствует о том, что рассматриваемое состояние квантовой системы является четным, $u$ - нечетным.

Сравним полученный энергетический спектр с ранее полученными результатами в рамках теории функционала плотности (DFT). В статье [7], как отмечено выше, подчеркивается, что фуллерен $\mathrm{Au}_{42}$ имеет „скромную энергетическую щель“ между высшей занятой молекулярной орбиталью (НОМО) и низшей незанятой молекулярной орбиталью (LUMO) равную $0.4 \mathrm{eV}$ при BP86 и $0.9 \mathrm{eV}$ при В3LYP вычислениях. В статье [21] методом функционала релятивистской плотности получено, что ширина щели между HOMO и LUMO coставляет $0.417 \mathrm{eV}$, отмечено, что ,этот результат близок к расчетным результатам [7]“. При этом согласно [7] HOMO имеет кратность вырождения пять, а LUMO кратность вырождения 4. Из анализа данных табл. 1 (стр. 3698 работы [7]) следует, что НОМО является орбиталь $h_{g}$ с десятью электронами, LUMO - opбиталь $g_{g}$ с количеством электронов, равным нулю в основном состоянии. Конфигурацию занятых электронами в основном состоянии орбиталей можно записать в виде $\left(a_{g}\right)^{2}\left(t_{1 u}\right)^{6}\left(h_{g}\right)^{10}\left(t_{2 u}\right)^{6}\left(g_{u}\right)^{8}\left(h_{g}\right)^{10}-42$ электрона занимают 6 нижних орбиталей. Если исходить из такого предположения, то энергетическая щель между НОМО и LUMO согласно спектру на рис. 3 была бы равной $0.483 \mathrm{eV}$.

Но на самом деле все не так просто. Дело в том, что рассматриваемая нами квантовая система в виде фуллерена $\mathrm{Au}_{42}$ является системой с сильными корреляциями. Особенностью сильнокоррелированной системы является то, что нижняя хаббардовская подзона в основном состоянии должна быть заполнена полностью, если на 42 узла фуллерена $\mathrm{Au}_{42}$ приходятся 42 электрона. Таким образом, роль НОМО играет пятикратно вырожденный уровень энергии $h_{u}$ нижней хаббардовской подзоны, роль LUMO - однократно вырожденный уровень энергии $a_{g}$ верхней хаббардовской подзоны, ширина энергетической щели равна $0.574 \mathrm{eV}$. Молекула фуллерена из сорока двух атомов золота находится в полупроводниковом состоянии, так же как и молекулы фуллерена $\mathrm{Au}_{16}-\mathrm{Au}_{20}[22,23]$ и нанотрубки из атомов золота малых размеров [24-27].

На следующем рис. 4 приведен для сравнения энергетический спектр фуллерена $\mathrm{Au}_{32}$ [28]. Сравнение энергетических спектров показывает, что порядок заполнения восьми нижних уровней, начиная с орбитали $a_{g}$ и заканчивая орбиталью $t_{1 u}$ нижней хаббардовской подзоны, 


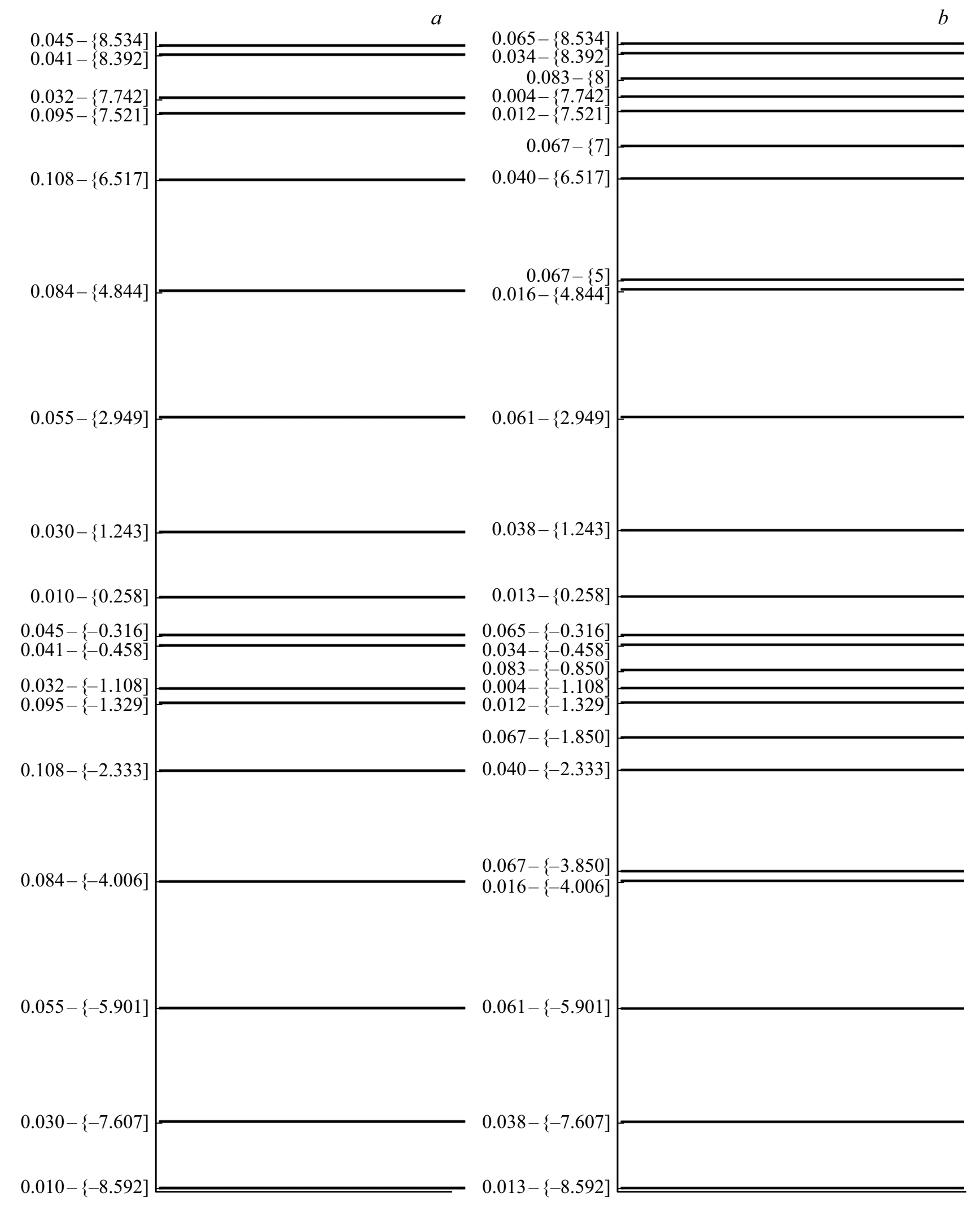

Рис. 2. $a-$ уровни энергии для атома № 1 в центре пятиугольника при значении параметров $\varepsilon=-2.85 \mathrm{eV}, B=-1 \mathrm{eV}$, $U=8.85 \mathrm{eV} ; b-$ уровни энергии для атома № 2 в центре шестиугольника при значении параметров $\varepsilon=-2.85 \mathrm{eV}, B=-1 \mathrm{eV}$, $U=8.85 \mathrm{eV}$. 


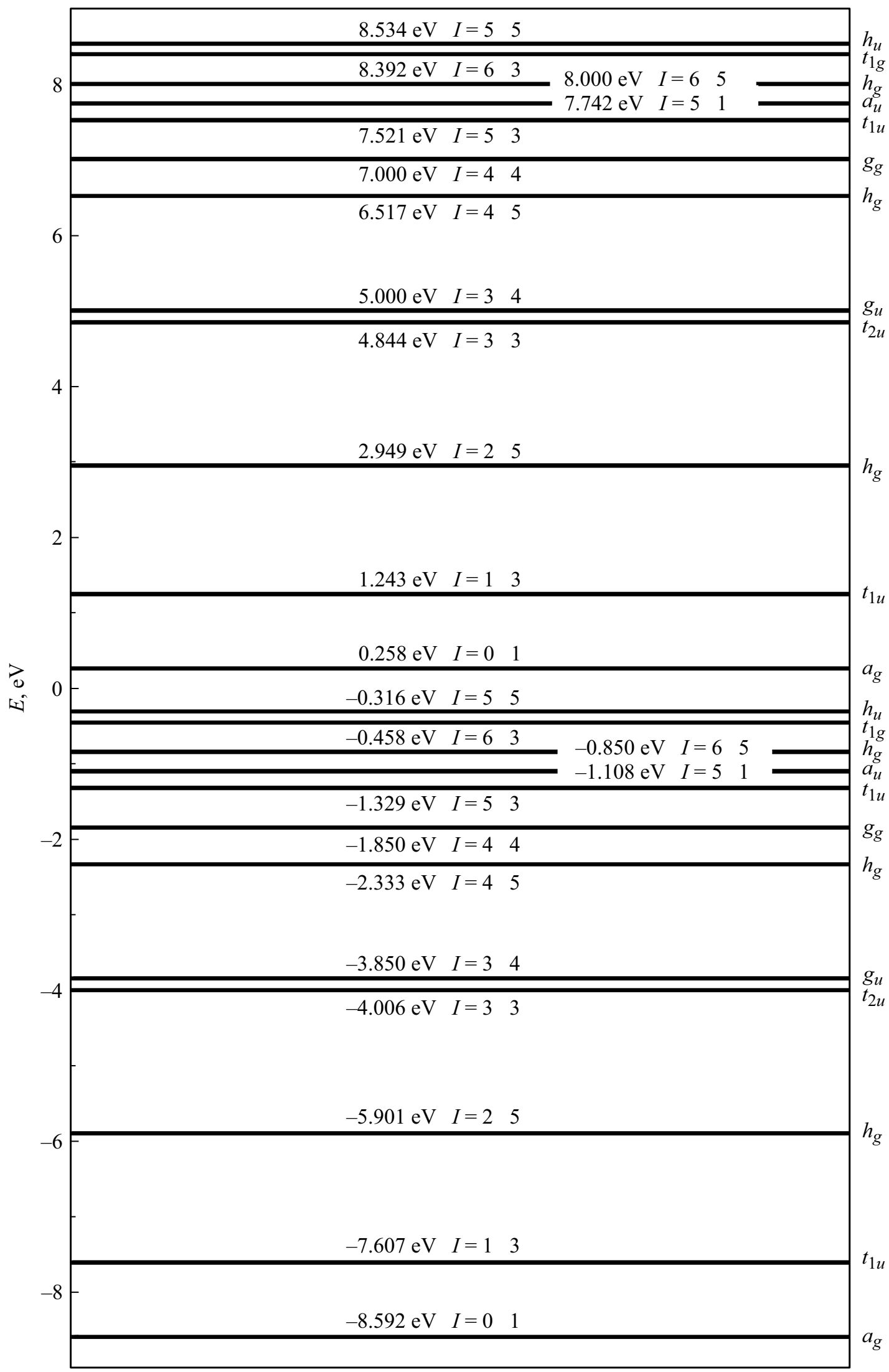

Рис. 3. Энергетический спектр молекулы фуллерена $\mathrm{Au}_{42}$ при следующих значениях параметров $\varepsilon=-2.85 \mathrm{eV}, B=-1 \mathrm{eV}$, $U=8.85 \mathrm{eV}$. 


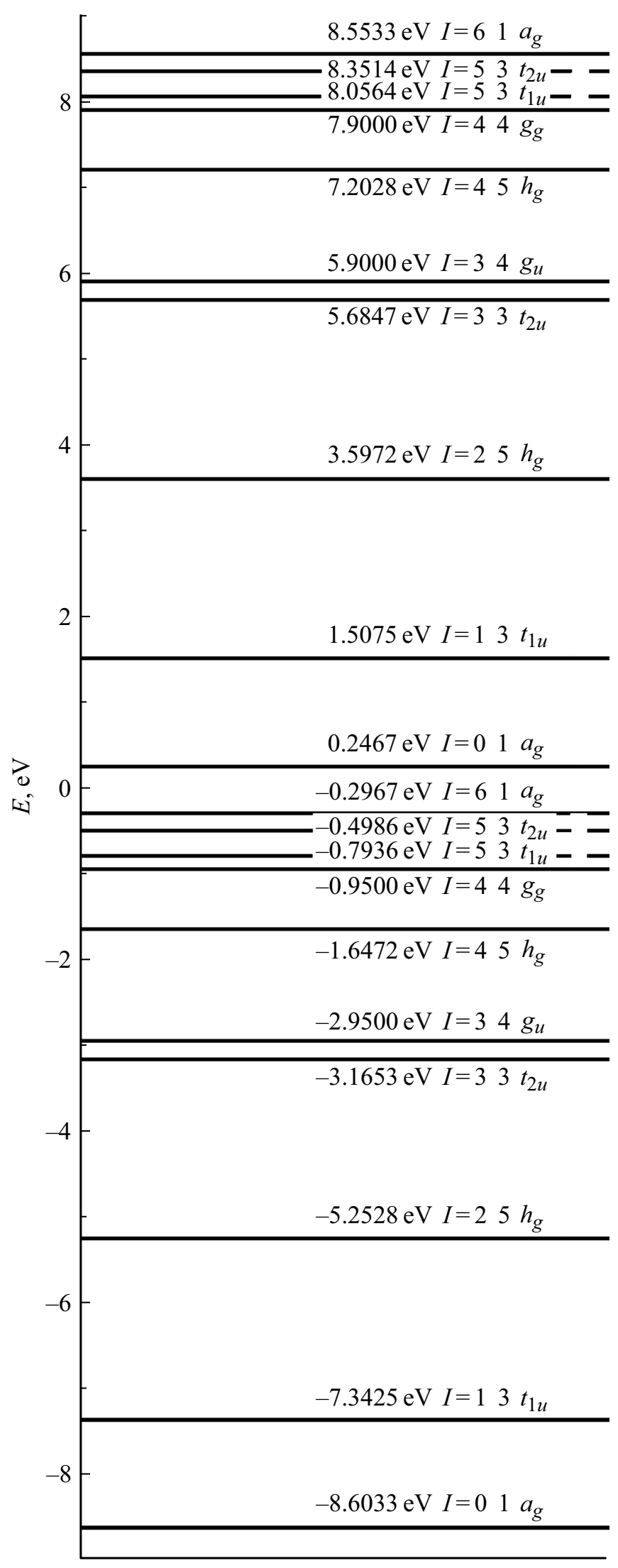

Рис. 4. Энергетический спектр молекулы фуллерена $\mathrm{Au}_{32}$ при следующих значениях параметров $\varepsilon=-2.85 \mathrm{eV}, B=-1 \mathrm{eV}$, $U=8.85 \mathrm{eV}$.

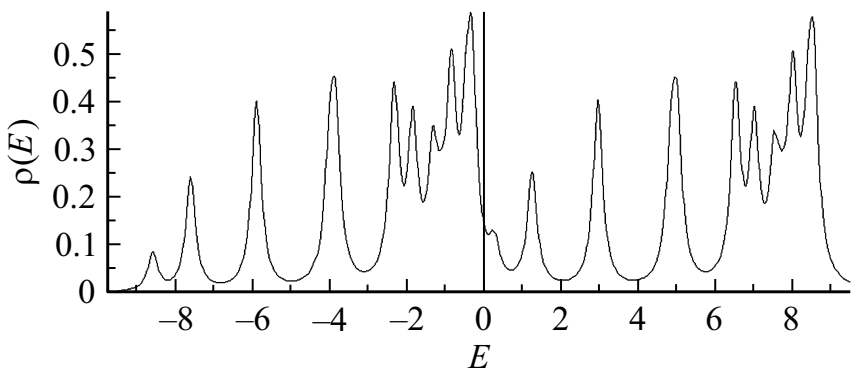

Рис. 5. Плотность электронного состояния в произвольных единицах при следующих значениях параметров: $U=8.85 \mathrm{eV}$, $B=1 \mathrm{eV}, \varepsilon=-2.85 \mathrm{eV}, C=0.15 \mathrm{eV}$.

происходит одинаково, одинаков также порядок расположения этих уровней энергий как в случае нижней подзоны, так и в случае верхней подзоны. Различие появляется на более высоких орбиталях, когда еще до полного заполнения подуровней энергии с орбитальным квантовым числом пять более выгодным оказывается заполнение двух подуровней энергии с орбитальным квантовым числом шесть, после чего заполняется третий подуровень энергии с $l=5$. Такого рода особенности проявляются при переходе от сферической симметрии к икосаэдрической [29].

Представляет интерес посмотреть, как плотно расположены уровни энергии на энергетическом спектре фуллерена $\mathrm{Au}_{42}$. Имея функции Грина (5), (6) можно вычислить плотность электронных состояний, она представлена на рис. 5. При моделировании дельта-функция представлена в виде: $\delta(E-x)=(1 / \pi) C /\left(C^{2}+(E-x)^{2}\right)$. Полуширина $C$ взята равной $0.15 \mathrm{eV}$. Пики плотности состояний электронов соответствуют сингулярностям Ван-Хова. Величина энергетической щели между нижней сингулярностью зоны проводимости (верхней хаббардовской подзоны) и верхней сингулярностью валентной зоны (нижней хаббардовской подзоны) является важным параметром плотности состояний электронов и в случае полупроводников совпадает с минимальной оптической щелью $[15,16]$. Анализ графика на рис. 5 показывает, что плотность электронных состояний больше в области НОМО-НОМО-3 зоны валентных электронов (вблизи фермиевской энергии) и меньше в области LUMO-LUMO+1 зоны проводимости.

На рис. 6 приведен спектр оптического поглощения золотого фуллерена $\mathrm{Au}_{42}$. На графике приведены значения пиков в электронвольтах, вблизи которых формируются полосы оптического поглощения.

На следующем рис. 7 приведена часть спектра поглощения для электрически отрицательно заряженного иона $\mathrm{Au}_{42}^{-}$, находящегося левее спектра на рис. 6. При этом для плотностей оптического поглощения дополнительно появятся полосы поглощения вблизи значений энергий 0.985 и $1.677 \mathrm{eV}$, которые по сравнению с полосами поглощения нейтрального фуллерена $\mathrm{Au}_{42}$ сме- 
щены в сторону инфракрасной области спектра, полосы находятся в ближней инфракрасной области спектра.

Первоочередной интерес для прикладных целей вызывает оптическое поглощение в ближней инфракрас-

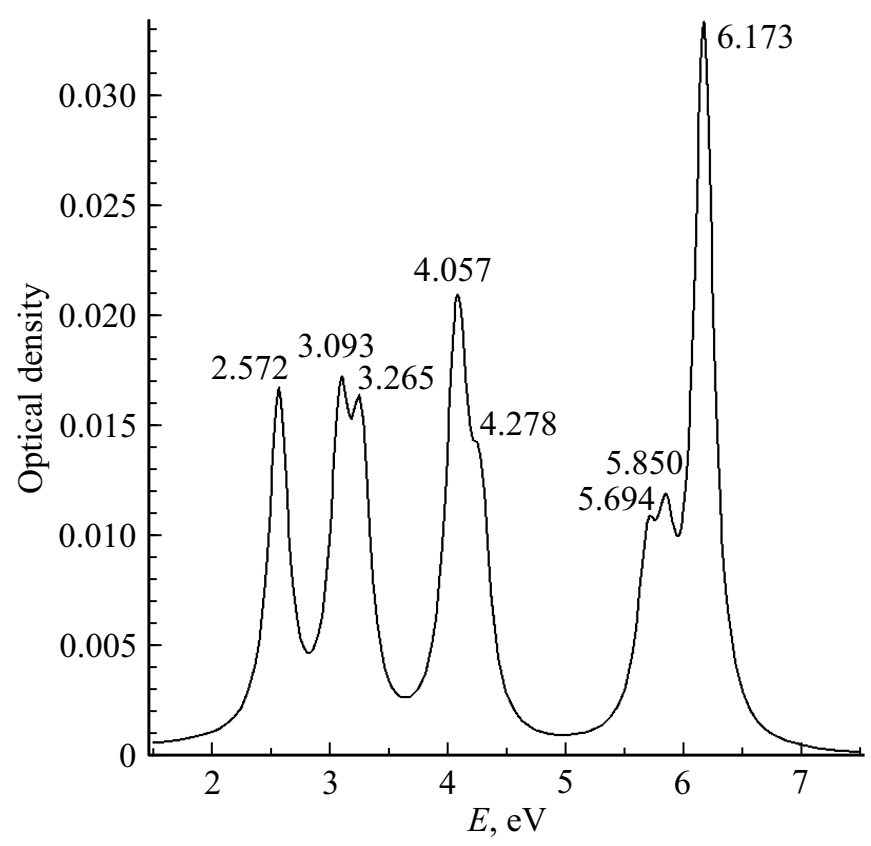

Рис. 6. Спектр оптического поглощения молекулы золотого фуллерена $\mathrm{Au}_{42}$ при следующих значениях параметров: $U=8.85 \mathrm{eV}, B=-1 \mathrm{eV}, \varepsilon=-2.85 \mathrm{eV}, C=0.15 \mathrm{eV}$.

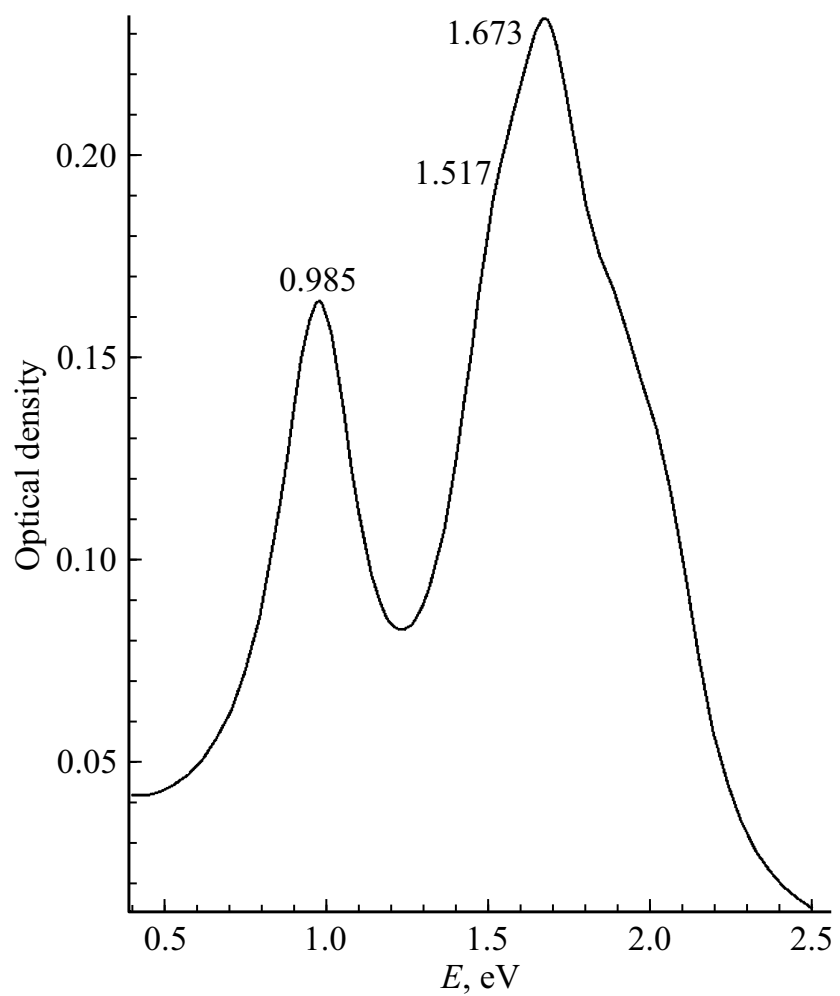

Pис. 7. Спектр оптического поглощения молекулы иона золотого фуллерена $\mathrm{Au}_{42}^{-}$при следующих значениях параметров: $U=8.85 \mathrm{eV}, B=-1 \mathrm{eV}, \varepsilon=-2.95 \mathrm{eV}, C=0.15 \mathrm{eV}$. ной области спектра. Дело в том, что излучение, соответствующее ближней инфракрасной области, пронизывает мягкие ткани и кровь живого организма, в частности человека, без сильного поглощения ими излучения [30,31]. Эксперименты свидетельствуют, что золотые фуллерены, в общем случае полые золотые клетки, при внедрении в организм аккумулируются на поверхности раковых клеток, сродство золотых фуллеренов к раковым клеткам выше сродства к здоровым клеткам [32-34]. Если после аккумуляции золотых фуллеренов на клетках злокачественной опухоли произвести инфракрасное излучение, то волны, соответствующие резонансным пикам слева, легко пройдут через мягкие ткани и кровеносные сосуды и достигнут золотых фуллеренов, внедренных в организм. После поглощения инфракрасного излучения фуллереном излучение преобразуется в тепло, которое передастся окружающим клеткам живой ткани, которые быстро нагреваются и расширяются, что приводит к образованию акустической волны в ультразвуковой части спектра. Сверхчувствительные ультразвуковые приемники фиксируют эту акустическую волну. В итоге на экране компьютера фотоакустического томографа возникнет высококонтрастное изображение злокачественной опухоли. Так с помощью золотых фуллеренов можно произвести фотоакустическую визуализацию злокачественного новообразования на ранней стадии заболевания.

Отметим, что сканирование с помощью метода магниторезонансной томографии ограничено количеством резонирующих ядер и позволяет обнаруживать опухоли размером более нескольких миллиметров или приблизительно 10 миллионов опухолевых клеток, что означает, что опухоли обнаруживаются только тогда, когда они достигают определенного порога. Тогда как фотоакустическая визуализация с помощью золотых фуллеренов как контрастных агентов является методом, который может помочь обнаружить опухоли на более ранней стадии ракового заболевания, что очень важно, поскольку развитие ракового заболевания происходит очень быстро.

Таким образом, исследуемые золотые фуллерены можно использовать как новый класс материалов для получения контрастных улучшений при диагностике раковых заболеваний на ранних этапах. После подтверждения диагноза эти же ионы фуллерена из атомов золота можно использовать при лечении рака - направив инфракрасный лазер к месту дислокации недоброкачественного новообразования, вызываем поглощение инфракрасного излучения фуллеренами из атомов золота. Вследствие безизлучательной релаксации электронов фуллерен нагревается и нагревает клетки опухоли, при температуpe выше $42^{\circ} \mathrm{C}$ раковые клетки начинают разлагаться с последующим исчезновением недоброкачественного новообразования. 


\section{4. Заключение}

Таким образом, в рамках весьма простой модели удается описать свойства молекулы икосаэдрического фуллерена из 42 атомов золота, получить аналитические выражения для Фурье-образов антикоммутаторных функций Грина, описывающих физико-химические свойства золотого нанокластера. Спектр элементарных возбуждений золотого фуллерена свидетельствует о наличии поглощения электромагнитных волн в ближней инфракрасной области спектра. Это свойство золотого фуллерена можно применять в тераностике раковых заболеваний.

\section{Конфликт интересов}

Автор заявляет об отсутствии конфликта интересов.

\section{Список литературы}

[1] X. Zhang, S. Wang, Y. Liu, L. Li, C. Sun. APL Mater. 5, 053501 (2017).

[2] P. Gruene, D.M. Rayner, B. Redlich, A.F.G. van der Meer, J.T. Lyon, G. Meijer, A. Fielicke. Science 321, 674 (2008).

[3] F. Baletto, R. Ferrando. Phys. Chem. Chem. Phys. 17, 28256 (2015).

[4] P. Singh, S. Pandit, V.R.S.S. Mokkapati, A. Garg. Int. J. Mol. Sci. 19, 1979 (2018).

[5] A. Zhang, W. Guo, Y. Qi, J. Wang, X. Ma, D. Yu. Nanoscale Res. Lett. 11, 279 (2016).

[6] W. Li, P.K. Brown, L.V. Wang, Y. Xia. Contrast Media Mol. Imaging. 6, 370 (2011).

[7] Y. Gao, C. Zeng. J. Am. Chem. Soc. 127, 3698 (2005).

[8] J. Wang, H. Ning, Q. Ma, Y. Liu, Y. Li. J. Chem. Phys. 129, 134705 (2008).

[9] D. Wang, X. Sun, H. Shen, D. Hou, Y. Zhai. Chem. Phys. Lett. 457, 366 (2008).

[10] F.C. Ju, M.R. Ping. Solid State Commun. 288, 53 (2019).

[11] A.D. Becke. Phys. Rev. A 38, 6, 3098(1988).

[12] C. Lee, W. Yang, R.G. Parr. Phys. Rev. B 37, 2, 785 (1988).

[13] M.P. Johansson, D. Sundholm, J. Vaara. Angew. Chem. Int. Ed. 43, 20, 2678 (2004).

[14] C. Adamo, V. Barone. J. Chem. Rev. 110, 13, 6158 (1999).

[15] П.Н. Дьячков. Журн. неорган. химии 60, 8, 1045 (2015).

[16] Г.И. Миронов. Журн. неорган. химии 63, 1, 72 (2018).

[17] J. Hubbard. Proc. Roy. Soc. A 276 (1365), 238 (1963).

[18] S.P. Shubin, S.V. Wonsowskii. Proc. Roy. Soc. A 145, 854, 159 (1934).

[19] Г.И. Миронов. ФТТ 50, 1, 182 (2008).

[20] Г.И. Миронов. ФММ 105, 4, 355 (2008).

[21] H. Ning, J. Wang, Q.-M. Ma, H.-Y. Han, Y. Liu. J. Phys. Chem. Solids 75, 5, 696 (2014).

[22] Г.И. Миронов. ФНТ 45, 4, 467 (2019).

[23] Г.И. Миронов. ФТТ 61, 6, 1204 (2019).

[24] Е.Р. Филиппова, Г.И. Миронов. ФНТ 37, 6, 644 (2011).

[25] Г.И. Миронов, Е.Р. Филиппова. ФТТ 54, 8, 1600 (2012).

[26] Г.И. Миронов, Е.Р. Филиппова. ФММ 113, 1, 11 (2012)

[27] Г.И. Миронов, Е.Р. Созыкина. ФНТ 45, 1, 128 (2019).

[28] Г.И. Миронов. Журн. неорган. химии 64, 10, 1064 (2019).
[29] E. Kaxiras. Atomic and Electronic Structure of Solids. Cambridge Univ. Press. Cambridge (2003). 676 p.

[30] J. Lee, D.K. Chatterjee, M.H. Lee et all. Cancer Lett. 347, 1, 46 (2014).

[31] J.F. Hainfeld, L. Lin, D.N. Slatkin, F.A. Dilmanian, T.M. Vadas, H.M. Smilowitz. Nanomedicine 10, 8, 1609 (2014).

[32] Y. Xia, W. Li, C. Cobley, J. Chen, X. Xia, Q. Zhang, M. Yang, E.C. Cho, P.B. Jarreau. Accounts Chem. Res. 44, 10, 914.(2011).

[33] B. Pang, X. Yang, Y. Xia. Nanomedicine 11, 13, 1715 (2016).

[34] J. Cuo, K. Rahme, Y. He, L. Li, J.D. Holmes, C.M. O’Driscoll. Int. J. Nanomedicine 12, 8, 6131 (2017).

Редактор К.В. Емщев 